\title{
BMJ Open Fructosamine measurement for diabetes mellitus diagnosis and monitoring: a systematic review and meta-analysis protocol
}

\author{
Jobert Richie N Nansseu, ${ }^{1,2}$ Joël Fokom-Domgue, ${ }^{3}$ Jean Jacques N Noubiap, ${ }^{4}$ \\ Eric V Balti, ${ }^{5,6,7}$ Eugène Sobngwi, ${ }^{5,8}$ André Pascal Kengne ${ }^{9}$
}

To cite: Nansseu JRN, Fokom-Domgue J, Noubiap JJN, et al. Fructosamine measurement for diabetes mellitus diagnosis and monitoring: a systematic review and meta-analysis protocol. BMJ Open 2015;5:e007689. doi:10.1136/bmjopen-2015007689

- Prepublication history and additional material for this paper is available online. To view these files please visit the journal online (http://dx.doi.org/10.1136/ bmjopen-2015-007689).

Received 15 January 2015 Revised 22 April 2015 Accepted 23 April 2015

\section{CrossMark}

For numbered affiliations see end of article.

\section{Correspondence to}

Dr Jobert Richie N Nansseu; jobertrichie_nansseu@yahoo. $\mathrm{fr}$

\begin{abstract}
Introduction: Fructosamine is a marker of glucose control reflecting the average glycaemic level over the preceding 2-3 weeks. Fructosamine has not gained as much popularity as glycated haemoglobin ( $\mathrm{HbA1C}$ ) for diabetes mellitus (DM) control monitoring, and the related underlying reasons remain unclear. We aim to search for and summarise available evidence on the accuracy of fructosamine measurements to diagnose and monitor DM.
\end{abstract}

Methods and analysis: This systematic review will include randomised control trials, controlled beforeand-after studies, time series designs, cohort studies, case-control studies and cross-sectional surveys reporting the diagnosis and/or monitoring of DM (type 1 DM, type 2 DM and gestational DM) with fructosamine compared with other measures of glycaemia (fasting glucose, oral glucose tolerance test, random glucose, $\mathrm{HbA1c}$ ), without any language restriction. We will perform electronic searches in PubMed, Scopus and other databases, supplemented with manual searches. Articles published from 1 January 1980 to 30 June 2015 will be eligible for inclusion in this review. Two authors will independently screen, select studies, extract data and assess the risk of bias with discrepancies resolved by consensus. We will assess clinical heterogeneity by examining the types of interventions and outcomes in each study, and pool studies judged to be clinically homogeneous. We will also assess statistical heterogeneity using the $\chi^{2}$ test of homogeneity and quantify it using the $\mathrm{I}^{2}$ statistic. Absolute accuracy measures (sensitivity, specificity) will be pooled in a bivariate random-effects model, allowing for intersetting variability. Negative and positive predictive values will be computed for fructosamine, compared with another measure of glycaemia from the pooled estimates of sensitivity and specificity, using Bayes' theorem.

Ethics and dissemination: This systematic review will use data from published studies and does not require ethics approval. Findings will be published in a peer-reviewed journal and presented at scientific conferences.

Trial registration number: PROSPERO (ID=CRD42015015930).

\section{Strengths and limitations of this study}

- To the best of our knowledge, this is the first systematic review and meta-analysis investigating fructosamine accuracy to diagnose and monitor diabetes mellitus, especially type 1 , type 2 and gestational diabetes mellitus.

- We will perform an exhaustive literature search to include all potential studies fulfilling the inclusion criteria.

- Likewise, we will use robust analytic methods to carefully examine the research questions with tools dedicated to the meta-analysis of diagnostic tests. We will also carefully assess risk of bias and clinical heterogeneity.

- Unfortunately, fructosamine measurement has not been standardised. There is no consensus on the method to use for its biochemical measurement or to assess its threshold values. It is possible therefore that some studies will lack the sensitivity, specificity, and positive and negative predictive values.

\section{INTRODUCTION}

\section{Rationale}

Diabetes mellitus (DM), particularly type 2 diabetes mellitus (T2DM), has reached epidemic proportions worldwide, fuelled by population growth, ageing, urbanisation, and increasing prevalence of obesity and physical inactivity. Estimates from the International Diabetes Federation indicate that the number of adults with $\mathrm{DM}$ in the world will expand by $55 \%$, from 381.8 million in 2013 to 591.9 million in 2035. ${ }^{1} \mathrm{DM}$ causes significant morbidity, disability and premature mortality through microvascular and macrovascular complications such as cerebrovascular disease, retinopathy, coronary heart disease, peripheral artery disease, nephropathy and neuropathy. ${ }^{1}$ Nevertheless, there is a body of evidence demonstrating that the onset and progression of DM complications can be prevented or delayed by achieving 
and maintaining near-normal metabolic control of the disease. ${ }^{2-4}$ Accordingly, tight metabolic control is the basis of contemporary diabetes management. For this, simple, reliable, affordable and easily reproducible tests are needed to monitor the outcomes of diabetes care, and to provide timely management feedback.

Glucose monitoring in diabetes focuses on acute as well as long-term changes. Acute term diabetes control monitoring has been traditionally based on fasting or postprandial blood glucose measurements, and provides useful information for daily adjustment of management strategies. Unfortunately, blood glucose concentrations fluctuate substantially and a single spot sample is insufficient to accurately characterise glycaemic control status, and implementing multiple blood glucose testing appears to be cumbersome to patients. Long-term diabetes control builds on parameters of chronic hyperglycemia existing in the form of glucose non-enzymatically bound to naturally occurring proteins ${ }^{5}$ and that are less sensitive to daily fluctuation in blood glucose levels, thus obviating the need for overly frequent testing.

Fructosamine, discovered about 30 years ago, is a marker of glucose control reflecting the average glycaemic level over the preceding 2-3 weeks. ${ }^{6}$ Consequently, it may be more appropriate for monitoring early response to treatments. ${ }^{6}$ Fructosamine measurement is quick, technically simple, inexpensive, precise, fairly free of interferences, unaffected by red blood diseases and easily automated for use with microsample volumes. ${ }^{7-10}$ Therefore, fructosamine has been proposed as a suitable parameter to screen for diabetes during pregnancy, ${ }^{11}{ }^{12}$ in low-income countries, ${ }^{13}$ and in areas where high prevalence of sickle cell disease and sickle cell traits have been reported. ${ }^{1415}$ However, fructosamine has not gained as much popularity as glycated haemoglobin (HbA1c) for DM control monitoring, despite some reports claiming that fructosamine could outperform HbAlc. ${ }^{16} 17$

The current protocol is for a systematic review to assess the accuracy of fructosamine measurements to diagnose and monitor the management of type 1 DM (T1DM), T2DM and gestational DM (GDM). We are particularly interested in answering the following questions: (1) what is the performance of fructosamine measurement in diagnosing diabetes versus traditional measurements (fasting glucose, oral glucose tolerance test (OGTT), random glucose, HbAlc), (2) does point-of-care fructosamine measurement equally perform as laboratory-based measurement, (3) are baseline fructosamine levels associated with future occurrence of diabetes complications and (4) how does fructosamine compared with other measures of glycaemia (OGTT, fasting glucose, HbAlc, random glucose) predict future occurrence of diabetes complications?

\section{Objective}

The aim of this study is to conduct a systemic review and meta-analysis of studies published from 1980 to 2015, in order to assess the accuracy of fructosamine measurement for diagnosing DM (T1DM, T2DM and GDM), and for monitoring the management of the disease.

\section{METHODS AND ANALYSIS}

Eligibility criteria

Inclusion criteria

- We will include randomised controlled trials, controlled before-and-after studies, time series designs, cohort studies (either prospective or retrospective), case-control studies and cross-sectional surveys;

- The cross-sectional surveys require the prior investigation of the performance of fructosamine measurement in diagnosing DM (T1DM or T2DM or GDM) versus traditional measurements (fasting glucose, OGTT, random glucose, HbA1c);

- The follow-up studies need to have reported either the baseline fructosamine levels that are associated with future occurrence of diabetes complications, or fructosamine capacity to predict future occurrence of diabetes complications when compared with other measures of glycaemia;

- We will consider all published and unpublished studies in human subjects, reported from 1 January 1980 to 30 June 2015, without sex or origin (country) restriction, accounting for the change in diagnosis and control criteria for DM over time. No language restriction will be applied.

\section{Exclusion criteria}

The following studies will not be considered in the present review:

- Studies not performed in human subjects;

- Studies reported before 1 January 1980 and after 30 June 2015;

- Letters, reviews, commentaries and editorials;

- Studies lacking primary data and/or explicit method description;

- Duplicates; for studies published in more than one report, the most comprehensive and up-to-date version will be used.

\section{Information sources}

\section{Electronic databases}

We will perform electronic searches in PubMed, MEDLINE, EMBASE, Google Scholar, Scopus, Cochrane Central Register of Controlled Trials (CENTRAL), ISI Web of Science (Science Citation Index), OCLC (Paper First and Proceedings First), PAIS International Database (EBSCO), WHO Global Health Library and POPLINE. Information on unpublished or ongoing studies will be sought through the WHO International Clinical Trial Registry Platform, Clinicaltrials.gov, Pan African Clinical Trials Registry (PACTR), the WHO Global Infobase and the meta-Register of Controlled Trials (mRCT). 


\section{Searching other resources}

Manual searches will include scanning the reference lists of relevant studies, specialist journals and conference proceedings.

\section{Search strategy}

We will perform a comprehensive search of the peerreviewed and grey literature to identify all appropriate studies available from 1 January 1980 to 30 June 2015 that fulfil our inclusion criteria. Table 1 displays the PubMed search strategy. This strategy will be adapted as appropriate for other databases. The methods for this systematic review are developed according to the PRISMA Guidelines for Meta-Analyses and Systematic Reviews of Observational Studies. ${ }^{18} 19$

\section{Study records}

Data extraction and management

Two authors will independently extract data from included studies using a standardised data extraction form that will be developed for this review. For each study, we will collect general information (authors, year, country, geographic region, type of publication), study design and methodology, sample size, age range, the type of assay performed to measure fructosamine and whether it was albumin corrected, other tests of glycaemia, study findings and outcomes. From each study comparing fructosamine to other measures of glycaemia (fasting glucose, OGTT, random glucose, HbA1c), we will extract data on sensitivity, specificity, positive and negative predictive values, and other measures of predictive accuracy when available, or we will extract the data needed to estimate those performance measures. Additionally, we will extract data on standardisation,

\begin{tabular}{ll} 
Table 1 & PubMed search strategy \\
\hline Number & Query \\
\hline$\# 1$ & fructosamine [tw] OR glycated proteins [tw] OR \\
& glycated albumin [tw] \\
$\# 2$ & screening [MeSH] OR diagnosis [MeSH] \\
m3 & monitoring [tw] OR control [tw] OR follow-up [tw] \\
& OR management [tw] OR surveillance [tw] OR \\
& care [tw] \\
$\# 4$ & \#2 OR \#3 \\
$\# 5$ & \#1 AND \#4 \\
$\# 6$ & "type 1 diabetes mellitus" [tw] OR "type 1 \\
& diabetes mellitus" [MeSH] OR "T1DM" [tw] \\
"type 2 diabetes mellitus" [tw] OR "type 2 \\
diabetes mellitus" [MeSH] OR "T2DM" [tw] \\
"gestational diabetes mellitus" [tw] OR \\
"gestational diabetes mellitus" [MeSH] OR \\
"GDM" [tw] \\
"diabetes mellitus" [MeSH] OR "diabetes" [tw] \\
$\# 10$ & \#6 OR \#7 OR \#8 OR \#9 \\
$\# 11$ & \#5 AND \#10 \\
$\# 12$ & \#11 Limits: from 1980/01/01 to 2015/06/30, and \\
& studies done in Humans \\
\hline
\end{tabular}

accuracy and precision of the assay used in each study. The two authors will compare the extracted data and resolve discrepancies by discussion and consensus, or arbitration of a third author. Relevant missing data will be sought by contacting the corresponding authors of included studies.

\section{Selection process}

We will develop and pilot a study selection guide using the inclusion criteria described above to make sure that the criteria are clear and can be applied consistently by all review authors. Two authors will independently screen the titles and abstracts obtained from the searches, and retrieve the full text of records deemed potentially eligible by at least one of the two authors. The two authors will afterwards independently review the full text of each potentially eligible study, compare their results and resolve any discrepancy by discussion and consensus. If a decision is not reached, a third review author will be consulted.

\section{Data collection process}

Internationally approved methodology for data collection and analysis will be used based on the guidance of the Cochrane Handbook of Systematic Reviews for Interventions. ${ }^{20}$

\section{Risk of bias in individual studies}

Two reviewers will independently assess risk of bias in each included study using the QUADAS-2 tool for the quality assessment of diagnostic accuracy studies. ${ }^{21} \mathrm{We}$ will provide a thorough description of the missing data and dropouts for each included study, and the extent to which these missing data could have influenced the results of the study. The authors will compare their results, and resolve any differences by discussion and consensus. Risk of bias and quality scores will be presented in a table and a flow diagram.

\section{Data synthesis, including assessment of heterogeneity}

We will pool studies found to be clinically homogeneous through a random-effects meta-analysis. Clinical heterogeneity will be investigated by examining the design and setting (including geographic region), the type of assay used to measure fructosamine and whether the fructosamine was albumin corrected, standardisation, accuracy and precision of the assay used, and the types of interventions and outcomes in each study. Statistical heterogeneity will be investigated using the $\chi^{2}$ test of homogeneity on Cochrane's $Q$ statistic, ${ }^{22}$ and we will quantify any between-study heterogeneity using the $\mathrm{I}^{2}$ statistic. ${ }^{23}$ Absolute accuracy measures (sensitivity, specificity) will be pooled in a bivariate random-effects model, allowing for intersetting variability. ${ }^{24}$ We will jointly illustrate the absolute pooled sensitivity and specificity for fructosamine using Hierarchical Summary Receiver Operating Characteristic regression curves. ${ }^{25}{ }^{26}$ Negative and positive predictive values will be computed for 
fructosamine compared with another measures of glycaemia from the pooled estimates of sensitivity and specificity using Bayes' theorem. The pooled relative sensitivities and specificities of fructosamine compared with each of the other measures of glycaemia will be obtained from the pooled absolute accuracy measures assessed by a bivariate model with the method of moments, ${ }^{27}$ allowing for intersetting variability. If the included studies differ significantly in design, settings, outcome measures or otherwise, we will summarise the findings in a narrative format.

The MADA package of the statistical software $\mathrm{R}$ (The $\mathrm{R}$ Foundation for statistical computing, Vienna, Austria) will serve for bivariate meta-analysis of sensitivity and specificity, ${ }^{28}$ and the MVMETA package will be used for assessing pooled relative sensitivities and specificities. $^{27}$ Statistically significant results will be set at a p value $<0.05$.

\section{Meta-biases}

Publication bias will be assessed with funnel plots of the diagnostic ORs, complemented with the use of Egger's test of bias. Additionally, the trim-and-fill method will be applied to assess the impact of potential publication bias. $^{29}$

We will present a table of the main characteristics of included studies and a summary table for potentially eligible studies that were subsequently excluded, and reasons for exclusion. Findings will be reported by time period, to account for changes of the criteria for diagnosing and monitoring diabetes over time.

Methods, findings and implications of the findings of this systematic review will be reported according to the PRISMA guidelines, including the extended guidance on reporting equity-focused systematic reviews. ${ }^{18} 19$ This protocol has been presented with regard to the PRISMA-P 2015 guidelines, ${ }^{30}$ and registered with PROSPERO (ID=CRD42015015930).

\section{ETHICS AND DISSEMINATION}

This systematic review does not require ethical approval since it is based on published studies and not individual participant data. The findings of this systematic review are expected to have important implications for clinical practice and research. The review will shed light on the potential use of fructosamine to diagnose or monitor T1DM, T2DM and GDM, especially in resource-poor settings and in situations where HbAlc measurements are imprecise. ${ }^{9} 1013$ The findings of this systematic review will be published in a peer-reviewed journal and shared at relevant scientific conferences.

\footnotetext{
Author affiliations

${ }^{1}$ Sickle Cell Disease Unit, Mother and Child Centre, Chantal Biya Foundation, Yaoundé, Centre Region, Cameroon

${ }^{2}$ Department of Public Health, Faculty of Medicine, Biomedical Sciences of the University of Yaoundé I, Yaoundé, Centre Region, Cameroon

${ }^{3}$ Department of Obstetrics and Gynecology, Faculty of Medicine, Biomedical Sciences of the University of Yaoundé I, Yaoundé, Centre Region, Cameroon
}

${ }^{4}$ Department of Medicine, Groote Schuur Hospital and University of Cape Town, Cape Town, South Africa

${ }^{5}$ National Obesity Centre, Yaoundé Central Hospital, Yaoundé, Centre Region, Cameroon

${ }^{6}$ Diabetes Research Centre, Brussels Free University-VUB, Brussels, Belgium ${ }^{7}$ Department of Clinical Chemistry and Radio-immunology, University Hospital Brussels-UZ Brussel, Brussels, Belgium

${ }^{8}$ Department of Medicine and Specialties, Faculty of Medicine, Biomedical Sciences of the University of Yaoundé I, Yaoundé, Cameroon

${ }^{9}$ Non-communicable Disease Research Unit, South African Medical Research Council and University of Cape Town, Cape Town, South Africa

Contributors JRNN, APK and JJNN conceived and designed the protocol, and JRNN drafted the manuscript. JRNN, APK, JJNN, ES, JF-D and EVB critically revised the manuscript for methodological and clinical content. All authors approved the final version of the manuscript.

Funding This research received no specific grant from any funding agency in the public, commercial or not-for-profit sectors.

Competing interests None declared.

Provenance and peer review Not commissioned; externally peer reviewed.

Open Access This is an Open Access article distributed in accordance with the Creative Commons Attribution Non Commercial (CC BY-NC 4.0) license, which permits others to distribute, remix, adapt, build upon this work noncommercially, and license their derivative works on different terms, provided the original work is properly cited and the use is non-commercial. See: http:// creativecommons.org/licenses/by-nc/4.0/

\section{REFERENCES}

1. International Diabetes Federation (IDF). Diabetes atlas. 6th edn. Brussels: International Diabetes Federation, 2013

2. The Diabetes Control and Complications Trial (DCCT) Research Group. The effect of intensive treatment of diabetes on the development and progression of long-term complications in insulin-dependent diabetes mellitus. $N$ Engl J Med 1993:329:977-86.

3. UK Prospective Diabetes Study (UKPDS) Group. Intensive blood-glucose control with sulphonylurea or insulin compared with conventional treatment and risk of complications in patients with type 2 diabetes (UKPDS 33). Lancet 1998;352:837-53.

4. Grundy SM, Cleeman JI, Merz CN, et al. Implications of recent clinical trials for the National Cholesterol Education Program Adult Treatment Panel III guidelines. J Am Coll Cardiol 2004;44:720-32.

5. Day JF, Ingebretsen CG, Ingebretsen WR, et al. Non-enzymatic glycosylation of serum proteins and hemoglobin: response to changes in blood glucose levels in diabetic rats. Diabetes 1980;29:524-7.

6. Nagasaka Y, Fujii S, Yaga K, et al. Clinical application of measuring serum fructosamine as an index of glycemic control in diabetic patients. Bull Yamaguchi Med Sch 1988;35:59-62.

7. Baker J, Reid I, Holdaway I. Serum fructosamine in patients with diabetes mellitus. N Z Med J 1985;98:532-5.

8. Hindle EJ, Rostron GM, Clark SA, et al. Serum fructosamine and glycated haemoglobin measurements in diabetic control. Arch Dis Child 1986;61:113-17.

9. Dafallah $A A$, Eskandarani $\mathrm{H}$, Rehaimi $\mathrm{A}$, et al. Fructosamine in $\mathrm{HbS}$ and G6PD-deficient Saudi Arabs in the Eastern Province of Saudi Arabia. Br J Biomed Sci 1994;51:332-5.

10. Koga M, Hashimoto K, Murai J, et al. Usefulness of glycated albumin as an indicator of glycemic control status in patients with hemolytic anemia. Clin Chim Acta 2011;412:253-7.

11. Hughes PF, Agarwal M, Newman P, et al. An evaluation of fructosamine in screening for gestational diabetes mellitus. Diabet Med 1995;12:708-12.

12. Agarwal MM, Hughes PF, Punnose J, et al. Gestationaldiabetes screening of a multi-ethnic, high risk population using glycated proteins. Diabetes Res Clin Pract 2001;51:67-73.

13. Islam N, Akhter J, Kayani N, et al. Fructosamine: an alternative assessment of past glycaemic control in developing countries. $J$ Pak Med Assoc 1993;43:238-40.

14. World Health Organization. Sickle-cell anaemia: report by the secretariat. Geneva: World Health Organization. 
15. Aliyu ZY, Gordeuk V, Sachdev V, et al. Prevalence and risk factors for pulmonary artery systolic hypertension among sickle cell disease patients in Nigeria. Am J Hematol 2008;83:485-90.

16. Misciagna G, Logroscino G, De Michele G, et al. Fructosamine, glycated hemoglobin, and dietary carbohydrates. Clin Chim Acta 2004;340:139-47.

17. Rendell M, Paulsen R, Eastberg S. Clinicaluse and time relationship of changes in affinity measurement of glycosylated albumin and glycosylated hemoglobin. Am J Med Sci 1986;292:11-14.

18. Moher D, Liberati A, Tetzlaff J, et al. Preferred reporting items for systematic reviews and meta-analyses: the PRISMA statement. Ann Intern Med 2009;151:264-9.

19. Welch V, Petticrew M, Tugwell P, et al. PRISMA-Equity 2012 Extension: reporting guidelines for systematic review with a focus on health equity. PLoS Med 2012;9:e1001333.

20. Higgins JP, Green S, eds. Cochrane handbook for systematic reviews of interventions. Versions 5.1.0 [updated March 2011]. The Cochrane Collaboration, 2011.

21. Whiting PF, Rutjes AW, Westwood ME, et al. QUADAS-2: a revised tool for the quality assessment of diagnostic accuracy studies. Ann Intern Med 2011;155:529-36.

22. Higgins JP, Thompson SG. Quantifying heterogeneity in a meta-analysis. Stat Med 2002;21:1539-58.
23. Higgins JP, Thompson SG, Deeks JJ, et al. Measuring inconsistency in meta-analyses. BMJ 2003;327:557-60

24. Reitsma JB, Glas AS, Rutjes AW, et al. Bivariate analysis of sensitivity and specificity produces informative summary measures in diagnostic reviews. J Clin Epidemiol 2005;58:982-90.

25. Rutter CM, Gatsonis CA. A hierarchical regression approach to meta-analysis of diagnostic test accuracy evaluations. Stat Med 2001;20:2865-84.

26. Harbord RM, Deeks JJ, Egger M, et al. A unification of models for meta-analysis of diagnostic accuracy studies. Biostatistics 2007;8:239-51.

27. Gasparrini A, Armstrong B, Kenward MG. Multivariate meta-analysis for non-linear and other multi-parameter associations. Stat Med 2012;31:3821-39.

28. Doebler P, Holling H, Böhning D. A mixed model approach to meta-analysis of diagnostic studies with binary test outcome. Psychol Methods 2012;17:418-36.

29. Duval S, Tweedie R. Trim and fill: a simple funnel-plot-based method of testing and adjusting for publication bias in meta-analysis. Biometrics 2000;56:455-63.

30. Moher D, Shamseer L, Clarke M, et al. Preferred reporting items for systematic review and meta-analysis protocols (PRISMA-P) 2015 statement. Syst Rev 2015;4:1. 